\title{
Fenómeno de transporte de una Kinesina como motor browniano con movimiento tipo Hand-Over-Hand
}

\author{
A Kinesin Transport Phenomena as a Brownian Motor with Hand-Over-Hand \\ Movement Type
}

\author{
D. R. Avellaneda Bernal ${ }^{a, *}$ \\ F. Naranjo Mayorga ${ }^{b}$
}

Recepción: 18-jun-14

Aceptación: 25-jun-14

\begin{abstract}
Resumen
El estudio del mecanismo de locomoción en motores proteicos es un tema de actual interés, ya que ha permitido explicar diferentes funciones celulares y, a la vez, ha proporcionado la replicación de motores a escala nanométrica; dicho estudio implica la comprensión de fenómenos estocásticos a microescala en sistemas periódicos que presentan algún tipo de asimetría y que se encuentran fuera del equilibrio térmico, de tal manera que se estudia un sistema que está fuera del equilibrio térmico y que a su vez está inmerso en un universo con disipación y generación de ruido térmico blanco, lo que genera un movimiento dirigido y, como tal, el fenómeno de transporte, de modo que se modelan las condiciones de simetría o asimetría del sistema. Desde el descubrimiento del movimiento browniano se han llevado a cabo investigaciones más arduas de los procesos físicos que se pude interpretar bajo este fenómeno y bajo las leyes de la termodinámica. Desde el punto de vista microscópico, el movimiento térmico aleatorio domina este mundo y hace que las fluctuaciones térmicas den origen a movimientos brownianos y a motores brownianos.
\end{abstract}

Palabras clave: Dinámica de biosistemas, Fenómenos de transporte, Kinesina, Langevin, Motor browniano, Movimiento Hand-Over-Hand.

\begin{abstract}
The study of the proteinaceous engines locomotion' mechanism is a current topic of interest, because it allows to explain different cell functions and simultaneously provides engines replications at nanometer scale. This study involves the understanding of stochastic phenomena at microscale in periodic systems with any kind of asymmetry and that are outside of the thermal equilibrium. In this way one system being studied that is out of thermal equilibrium and at the same time, is immersed in a universe with dissipation and generation of white thermal noise, which generates a directed movement and as the transportation phenomenon does, so that the system symmetry or asymmetry conditions are modeled. Since the discovery of Brownian motion, there have been carried out some of the most arduous investigations of the physical
\end{abstract}

\footnotetext{
${ }^{a}$ Universidad Federal Rural de Pernambuco, UFRPE, Brasil.

*Autor de correspondencia: davidave16@gmail.com

${ }^{\mathrm{b}}$ Grupo de Física Teórica y Computacional, Universidad Pedagógica y Tecnológica de Colombia, Tunja, Colombia.
} 
processes, that can be interpreted under this phenomenon and under the thermodynamics laws. From the microscopic point of view, the random thermal motion dominates this world and makes thermal fluctuations give rise to Brownian motion and Brownian motors.

Key words: Brownian Motors, Biosystems Dynamics, Kinesin, Langevin, Movement Hand-Over-Hand, Transport Phenomena.

\section{Introducción}

Se estudia un sistema que está fuera del equilibrio térmico y, a su vez, inmerso en un universo con disipación y generación de ruido térmico blanco, lo que genera un movimiento dirigido y, como tal, el fenómeno de transporte; de modo que se estudian y modelan las condiciones de simetría o asimetría del sistema.

Estos sistemas pueden presentarse tanto macroscópica como microscópicamente. Un sistema a nivel macroscópico, en el que se observaría dicho fenómeno de transporte, se puede presentar, por ejemplo, en un molino de viento o en un reloj de cuerda automática, mientras que en un sistema a nivel microscópico, en donde el ruido térmico es importante.

Para que el sistema permanezca en constante movimiento es necesario mantenerlo alejado del equilibrio termodinámico, y así poder utilizar esa energía de no-equilibrio para lograr, junto con la asimetría del sistema, un movimiento dirigido; este fenómeno de transporte es conocido como Efecto Ratchet. A nivel microscópico existen máquinas que pueden operar en presencia de movimiento térmico, como, por ejemplo, los motores moleculares o sistemas con comportamiento Ratchet; por esta razón, el concepto de motor browniano se utiliza a menudo para los motores tipo biológico y molecular.

En el desarrollo del trabajo se utilizó un potencial del tipo Pulsating Ratchets, más conocido como Flashing ratchet; en este tipo, las partículas quedan atrapadas en un potencial periódico asimétrico de dientes de sierra, el cual puede ser encendido o apagado; cuando el potencial se apaga, la difusión se realiza al azar, y cuando se enciende se convierte en un movimiento neto dirigido. Este movimiento dirigido puede convertirse en nanomáquinas encargadas del transporte de seres unicelulares o del acarreo de ingredientes en el interior de una célula.

Teniendo en cuenta este comportamiento, estudiamos el fenómeno para sistemas lejos del equilibrio, con el modelamiento de las ecuaciones de Langevin y Fokker-Planck, con el fin realizar una mímica del desplazamiento de una kinesina sobre un microtúbulo. Así mismo, se deben comprender los fenómenos de fluctuación-disipación y, posteriormente, la rueda dentada descrita por Feynman en sus lecturas [1], para así tener una comprensión del efecto Ratchet.

Gracias a la amplia investigación acerca de los fenómenos de transporte, se han generado gran cantidad de modelos físicos y matemáticos que tratan de explicarlos, de modo que adoptaremos uno: el propuesto por J. J. Mazo et al. [2], de la kinesina con movimiento Hand-over-Hand sobre un microtúbulo, modelando la ecuación de Langevin del desplazamiento de una partícula browniana con un potencial tipo Pulsating y con ruido térmico blanco de media nula.

\section{Metodología}

Basados en Mazo et al. [2], se realizó una completa revisión bibliográfica que involucró a [3-16], para analizar el tipo de movimiento con mayor probabilidad de ocurrir a nivel microscópico, de la proteína kinesina mientras se desplaza por un microtúbulo en el interior de una célula [18-21].

\subsection{Fluctuación disipación de una partícula browniana}

Al tomar una partícula de masa m, cuya coordenada del centro de masas en el tiempo $t$ se designa por $x(t)$, y que a su vez está inmersa en un fluido a temperatura $T$, estará gobernada por la siguiente ecuación de movimiento:

$$
m \ddot{x}(t)+V^{\prime}(x(t))=-\eta \dot{x}(t)+\xi(t)
$$

A partir de esta y junto con la ley de Stokes, obtener un flujo de partículas,

$$
J=-\frac{\rho F}{6 \pi v r}
$$

con la ley de Fick, obtener una difusión,

$$
D=\frac{\kappa T}{6 \pi v r}
$$


Y con un tratamiento matemático obtener información del desplazamiento cuadrático medio para intervalos de tiempo $\tau$, en función de $\kappa$ y de $T$,

$$
\left\langle x^{2}\right\rangle=\frac{\kappa T}{3 \pi v r} \tau
$$

Esta ecuación puede ser obtenida a partir de (1), con un tratamiento en el cuál se involucra el potencial químico; a su vez, también puede ser obtenida a partir de un tratamiento estadístico de la ecuación de Langevin sin fluctuación externa.

\subsection{Ecuación de Fokker-Planck}

La ecuación de Fokker-Planck está dada por:

$$
\frac{\partial P}{\partial s}=-\frac{\partial}{\partial v}\left(M_{1} P\right)+\frac{1}{2} \frac{\partial^{2}}{\partial v^{2}}\left(M_{2} P\right)
$$

la cual solo depende de los dos primeros momentos de la distribución; para el caso del fenómeno de transporte, es necesario obtener una distribución de posición.

Para una partícula browniana sometida a una fuerza externa $F_{e}$, esta ecuación queda definida por:

$$
\eta \partial_{t} \rho x(x, t)+\partial_{x}\left[F_{e}(x) \rho x(x, t)\right]-\kappa T \partial_{x}^{2}[\rho x(x, t)]=0
$$

También puede ser descrita por la ecuación de Langevin para un proceso estocástico $X(t)$ :

$$
\frac{d X}{d t}=f(X(t))+g(X(t)) \xi(t)
$$

con las siguientes funciones:

$$
\begin{aligned}
f(x) & =\frac{1}{\eta} F_{e}(x) \\
g(x) & =\frac{1}{\eta} \\
\sigma^{2} & =2 D \eta^{2}=\eta^{2} \kappa T
\end{aligned}
$$

\subsection{Efecto ratchet "rueda dentada"}

Feynman, en sus lecturas [1], explica cómo es posible obtener trabajo a partir de fluctuaciones aleatorias de un sistema de partículas inmersas en un depósito de calor que está fuera del equilibrio termodinámico y producir un movimiento direccionado; este movimiento tiene como base el movimiento browniano, y producirá un efecto llamado "efecto ratchet", que consiste en la aparición de una corriente dirigida o transporte de partículas en una dirección preferencial como consecuencia del efecto de una perturbación periódica o aleatoria (alejar el sistema del equilibrio termodinámico) de promedio nulo actuando sobre una o varias partículas brownianas inmersas en un sistema periódico en el espacio, pero asimétrico.

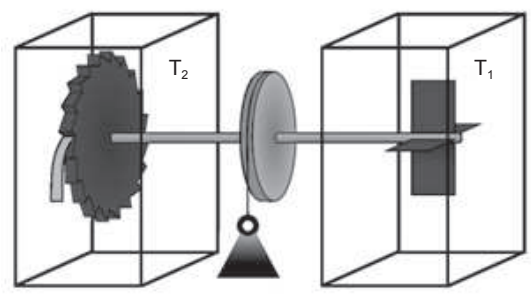

Figura 1. Rueda dentada y Trinquete.

\subsection{Fluctuating potential ratchet}

El flashing ratchet consiste en una o más partículas brownianas en una dimensión, sometidas a un potencial periódico en el espacio, pero asimétrico, que puede prenderse o apagarse a voluntad (estado del potencial en on o en off). Consideraremos la dinámica estocástica de la ecuación de Langevin, que viene de la ecuación generalizada de SmoluchowskiFeynman y junto con el potencial tipo flashing,

$$
\eta \dot{x}(t)=-V^{\prime}(x(t))[1+f(t)]+\xi(t)
$$

Donde $x(t)$ representa la posición de la partícula en el instante $t ; \dot{x}=\frac{d x}{d t} ; \eta$ es el coeficiente de fricción de la partícula, y $\zeta(t)$ es el ruido blanco gaussiano de media nula con correlación $\left(\xi(t) \xi\left(t^{\prime}\right)\right)=2 \eta \kappa T \delta(t-$ $\left.t^{\prime}\right)$. El potencial $V(x)$ es un potencial periódico de periodo $L$ y asimétrico, de modo que $-V^{\prime}(x)=-\frac{d V}{d x}$ es la fuerza aplicada debida al potencial que siente una partícula colocada en la posición $x$; por otro lado, la función $f(t)$ está restringida a dos valores \pm 1 , "on" u "off".

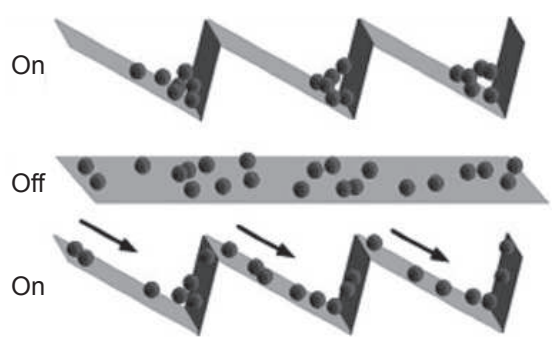

Figura 2. Potencial flashing ratchet en estado on-off-on.

$\mathrm{Al}$ analizar los casos en los que el potencial se enciende y apaga, obtendríamos que para ambos 
casos $\dot{x}=0$, lo que da a entender que la corriente de partículas es cero, $J(x)=0$, debido a que no existe transporte neto de partículas en ninguna de las dos direcciones, y, por tanto, el fenómeno de transporte se da en el transcurso en el que el potencial se enciende y se apaga, tal como lo muestra la distribución:

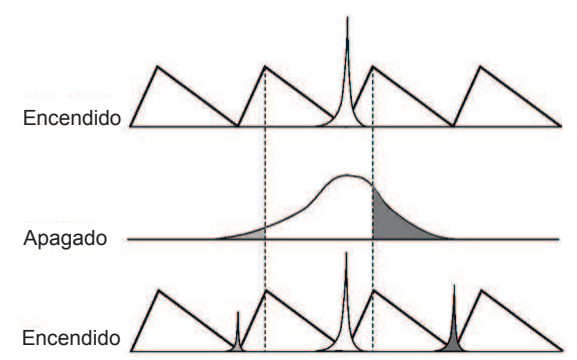

Figura 3. Flashing Ratchet. Muestra el potencial aplicado $V(x)$ para obtener un ratchet tipo flashing junto con la densidad de probabilidad de las partículas inmersas en este.

\subsection{Motores moleculares}

Los motores moleculares son máquinas biológicas a escala microscópica capaces de transformar o consumir el combustible químico y convertirlo en trabajo mecánico. Muchos motores moleculares a base de proteínas aprovechan la energía libre de productos químicos liberados por la hidrólisis de ATP para llevar a cabo algún tipo de movimiento o para generar un transporte proteico a nivel intracelular; todo esto ha despertado gran interés por el estudio de máquinas moleculares, el cual, además, ha sido impulsado con la observación y experimentación, puesto que, bajo diferentes técnicas de óptica microscópica, puede ser observado el comportamiento y la arquitectura molecular en el interior de la célula, y todo debido a que el acarreo de nutrientes depende del transporte activo y dirigido de macromoléculas, membranas o cromosomas en el citoplasma celular.

El interés por el fenómeno de transporte se debe también a las posibles aplicaciones; por ejemplo, los efectos perjudiciales de un trancón en el tráfico vehicular de una ciudad pueden ser generados por una irregularidad del flujo vehicular o por falencias en el transporte de estos, y comparado con la escala microscópica, las falencias en el transporte a nivel molecular pueden ser generadoras de diferentes enfermedades, que afectan la calidad de vida de los seres vivos; de tal forma que la comprensión del fenómeno de transporte molecular puede, eventualmente, ser de gran utilidad para curar estas enfermedades.
Por otro lado, parece que la naturaleza constituye los motores más potentes a partir de muchas subunidades pequeñas, lo más pequeñas posibles, denominadas motores moleculares, que se caracterizan por la cantidad de movimiento que pueden realizar debido a la inversión activa del flujo natural de algunos procesos químicos o mecánicos; estas son las máquinas de un solo tiempo y las máquinas cíclicas, entre las cuales se encuentran la miosina, la kinesina y las polimerasas, ya que son motores moleculares capaces de realizar un número ilimitado de pasos sin que cambie su estructura, siempre y cuando dispongan de cantidades suficientes de moléculas de combustible

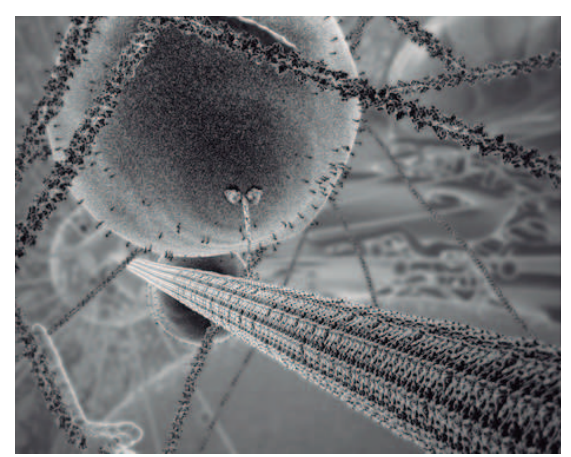

Figura 4. Esquema de una Kinesina transportándose a través de un microtúbulo y llevando consigo una vesícula transportadora (Tomada de [17]).

En este trabajo se muestra el modelamiento de la locomoción de la kinesina a través de un microtúbulo en el interior de una célula eucariota (aquellas que tienen un núcleo bien definido, lo que hace que el material genético hereditario se encuentre solamente en el núcleo, a diferencia de las procariotas, en las que se encuentra disperso en todo el citoplasma), transformando el combustible químico, la adenosín trifosfato o ATP en proceso de hidrolisis, en trabajo mecánico.

La kinesina (figura 4) es una proteína tipo MAP con actividad motriz; tiene un peso de $380 \mathrm{kDa}$ y unos $100 \mathrm{~nm}$ de largo; es un dímero formado por dos cadenas pesadas (que se entrecruzan en hélice y forman dos cabezas globulares), cada una de las cuales se une a una cadena ligera. En la kinesina se pueden distinguir dos dominios: cabeza y cola. La cabeza puede unirse a los microtúbulos del citoesqueleto y tiene actividad ATPasa, mientras que la cola se une a la membrana de naturaleza lipídica de las vesículas que, de este modo, pueden ser 
transportadas. El tipo de transporte puede llevarse a cabo de dos formas posibles [2]: transporte tipo hand-over-hand o transporte tipo inchworm. La velocidad del desplazamiento en el axón es de $2 \mu \mathrm{m} / \mathrm{s}$. - $3 \mu \mathrm{m} / \mathrm{s}$. y equivale a un transporte rápido ${ }^{1}$.

La kinesina se caracteriza por tener un transporte centrifugo o anterógrado, es decir, hacia la periferia celular o hacia la parte positiva (+) del microtúbulo en el axón de una neurona. En general, esta proteína es muy importante en todas las células para mantener el retículo endoplasmático alejado del centro celular e intervenir en el movimiento de los cromosomas durante la mitosis.

\section{Modelo físico}

Feynman modeló una máquina mecánica que funcionó sin violar la segunda ley de la termodinámica, y produjo una rectificación del movimiento y, por tanto, un efecto ratchet; ahora bien, para comprender lo nuevo, empezaremos considerando lo que ya se conoce, es decir, con una máquina mecánica macroscópica.

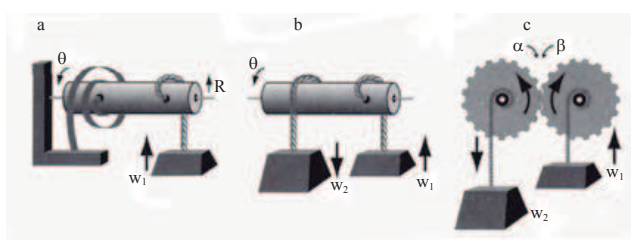

Figura 5. Tres máquinas mecánicas para el modelamiento de los motores moleculares (Tomada de [4]).

La figura 5(a) muestra una máquina de un solo tiempo: inicialmente la rotación del eje de radio $R$ en la dirección opuesta a la flecha almacena energía potencial en un muelle espiral; al liberar el eje, el muelle se desenrolla y aumenta $\theta$, generando un trabajo útil sobre una carga externa; la consideración importante es que en el momento en el que el muelle se desenrolla, la máquina se detiene. La figura 5(b) muestra un análogo cíclico de (a), aquí la máquina está comprendida por un eje central cuya fuente de energía es el peso $w_{2}$, el cual sube una carga externa $w_{1}$ (con las consideraciones pertinentes, es decir, $w_{2}>w_{1}$ ) contra su dirección natural de

\footnotetext{
${ }^{1}$ Los transportes proteicos en el axón de las neuronas puede dividirse en tres clases según la velocidad de transporte: un transporte lento $(1-5 \mathrm{~mm} /$ día $=1-4 \mu \mathrm{m} / \mathrm{s})$, uno rápido (50$200 \mathrm{~mm} /$ día $=0,5-3 \mu \mathrm{m} / \mathrm{s})$ y muy rápido $(1000-2500 \mathrm{~mm} /$ día $=$ $11-28 \mu \mathrm{m} / \mathrm{s})$.
}

movimiento. Aquí la máquina es capaz de convertir la disminución de energía potencial de su fuente en un aumento de la energía potencial de su carga. La máquina mostrada en la parte (c) de la figura 5 tiene dos ejes con posiciones angulares $\alpha$ y $\beta$, acoplados mediante engranajes (se considera que están a razón $1: 1$, es decir, por cada giro completo de $\alpha, \beta$ efectúa una revolución).

Enfocaremos toda nuestra atención en la tercera máquina, ya que junto con la suposición que daremos a continuación nos permitirá realizar un ajuste al modelo real del movimiento de la kinesina sobre un microtúbulo. Como esta máquina involucra dos engranajes, vale la pena destacar que en la mecánica estos pueden estar interactuando rígidamente. Una forma de interacción es respecto a las variaciones angulares de los engranajes, es decir, $\alpha=\beta$ (si están 1:1), pero podríamos imaginar unos engranajes de "goma" que pudieran deformarse y deslizarse uno con respecto al otro, bajo la acción de una diferencia de cargas pesadas. De esta forma, la relación se convertiría en $\alpha=\beta+2 \pi n / N$, con $N$ como el número de dientes, y $n$ un entero cualquiera.

Al realizar un gráfico hipotético de energía potencial o paisaje de energía interna del sistema, tal como se muestra en la figura 6 , podemos analizar un poco más su comportamiento. Las máquinas reales no son perfectas, irregularidades pueden darse en los engranajes y pueden ser representadas como las protuberancias en el paisaje de energía; así pues, los engranajes no giran libremente, aun cuando estén en uno de los valles (los valles del paisaje pueden llegar a ser regiones estables de la máquina), la consideración de engranajes de goma puede producir que se efectúe un deslizamiento y que a su vez suponga un salto de valle a valle cuya probabilidad será mayor en las protuberancias más bajas de las cordilleras que separan los valles.

Analicemos un fenómeno invisible: el hecho de considerar una fuerza impulsora grande en comparación con una fuerza de carga, es decir, $w_{1} \ll w_{2}$. Para este caso diseñaremos una máquina que resbala al valle contiguo más cercano, es decir, $\alpha$ disminuye $\sin$ que $\beta$ lo haga; este es un nuevo fenómeno que no se observa en la máquina de Feynman, y es el hecho de que cuando se consume energía impulsora no se realiza ningún trabajo útil, si no que se disipa debido al entorno viscoso. De esto podemos resumir que la 


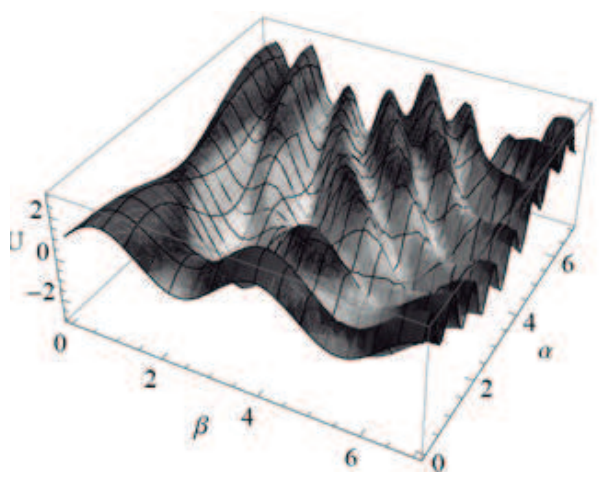

Figura 6. Paisaje de energía potencial hipotético para la máquina de engranajes del panel $\mathrm{c}$ de la figura 5 con algunas imperfecciones en los engranajes y sin algún tipo de carga. Consideremos ahora una diferencia entre la relación de cargas y mantengamos las irregularidades de los engranajes. En primer lugar, es necesario definir los signos de $\alpha$ y de $\beta$. Para $\alpha$, aumentará cuando el engranaje de la izquierda gire en el sentido de giro de las agujas del reloj, mientras que para $\beta$ aumentará cuando el engranaje derecho gire en contra del giro de las manecillas. De esta forma, el efecto impulsor que se genera debido a la diferencia de cargas producirá que se incline el paisaje en la dirección de $\alpha$ decreciente; por otro lado, la carga producirá inclinar el paisaje hacia arriba para los valores negativos de $\beta$, tal como se muestra en la figura 7.

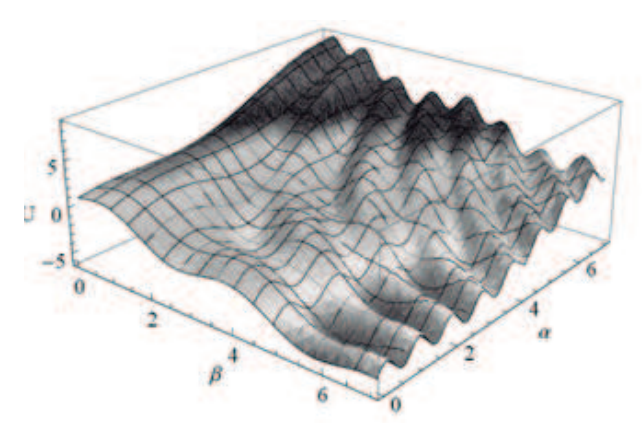

Figura 7. Paisaje de energía potencial hipotético para la máquina de engranajes de la figura 5 (c) con la configuración de cargas $w_{1}<w_{2}$, además de imperfecciones en la máquina como el hecho de tener engranajes de goma.

máquina deja de hacer trabajo útil cuando:

a. $w_{1}=w_{2}$, de manera que la máquina está en equilibrio mecánico (esto se reflejaría en el paisaje de energía, haciéndose horizontal), o bien si

b. la tasa de deslizamiento se hace demasiado grande.

Se puede resumir y concluir todo el análisis que hemos realizado a las máquinas y compararlo con el estudio del efecto ratchet, todo esto a nivel molecular, en lo siguiente:
1. Las máquinas de escala molecular se desplazan caminando aleatoriamente por su paisaje de energía libre, en vez de deslizarse determinísticamente.

2. Pueden atravesar barreras de energía potencial, con un tiempo medio de espera dado por un factor exponencial más conocido como factor de Boltzmann.

3. Pueden almacenar energía potencial (esto es, en parte, lo que da lugar al paisaje de energía), pero no energía cinética (porque la disipación viscosa es grande en el mundo nanoscópico).

4. Una máquina térmica puede convertir energía interna almacenada ( $\epsilon$ para el caso de Feynman) en movimiento direccional, si es estructuralmente asimétrica.

5. La asimetría estructural del sistema, por sí sola, no es suficiente para que esta avance, puesto que ella no es dirigida si está en equilibrio termodinámico. Para obtener trabajo útil es necesario llevarla fuera del equilibrio, lo que supone un paisaje de energía libre inclinado, como el de la figura 7.

\section{Resultados y discusión}

Para iniciar el análisis del movimiento de la kinesina, veamos la reacción que se produce en el sistema kinesina-microtúbulo. En la kinesina se encuentran una serie de proteínas catalizadoras de energía, llamadas enzimas, que actúan sobre unas moléculas denominadas sustratos (que se encuentran en los microtúbulos) y, como consecuencia, se convierten en moléculas diferentes denominadas productos (en este fenómeno no se conserva la sustancia original, se transforma su materia, manifiesta energía y es irreversible). Ahora bien, esperamos obtener un paisaje de energía libre de este sistema, y en él establecer un camino aleatorio unidimensional que descienda por los valles; esto correspondería al proceso sucesivo de sustrato que hace que descienda por los valores negativos de $\alpha$ (coordenada química) y combinado por los pasos espaciales sucesivos mostrados como los valores negativos de $\beta$ (coordenada mecánica). Si la concentración del producto se mantiene baja, el camino aleatorio a lo largo del eje de $\alpha$ tendrá un paso irreversible y se cumplirá con lo analizado anteriormente sobre el paisaje de energía inclinado de la figura 7 . 


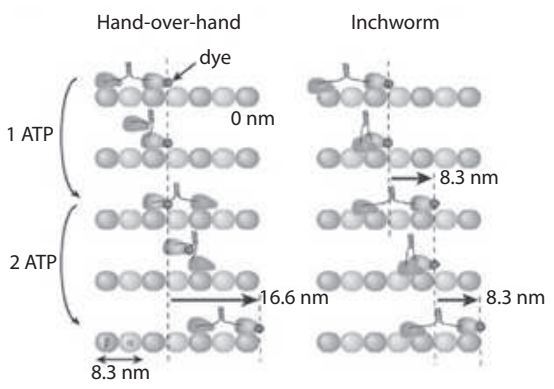

Figura 8. Representación de los tipos de movimiento de la máquina molecular dada por la kinesina transportándose en un microtúbulo. La figura de la izquierda muestra el caminar tipo Hand-over-hand, allí la kinesina tiene una periodicidad de $2 l_{0}$, mientras que en la imagen de la derecha muestra el tipo Inchworm, en el cual la kinesina tiene periodicidad $l_{0}$ (figura tomada de [19]).

Las propiedades estructurales del microtúbulo, junto con la química del sistema kinesinamicrotúbulo, forman un papel determinante para la dirección de movimiento de la kinesina; esto se ha podido comprobar experimentalmente [19-21] y ha arrojado una serie de resultados importantes que explican el fenómeno de transporte a nivel celular y a escala nanométrica, pero los resultados no son fáciles de analizar y no son concluyentes frente a la forma de como "caminan" estas proteínas en los microtúbulos. Por esta razón se propusieron dos formas posibles de caminar de la kinesina, como se muestra en la figura 8, la primera denominada movimiento tipo Hand-Over-Hand, y la segunda, movimiento tipo Inchworm.

Estudios experimentales [20,21] demostraron que la forma más probable del tipo de movimiento de la kinesina es la llamada hand-over-hand, de modo que se adopta este modelo, y así la ecuación que modelaría el sistema estaría dada por la ecuación de Langevin (8):

$$
\eta \dot{x}(t)=-V^{\prime}(x(t))[1+f(t)]+\xi(t)
$$

La energía potencial del sistema fue modelada considerando que las partículas (las dos cabezas de la kinesina) están interactuando bajo los efectos del potencial flashing, lo que genera dos partículas moviéndose en dos dimensiones; por tanto, la energía del sistema esta descrita por:

$$
V\left(x_{1}, x_{2}\right)=V_{1}\left(x_{1}, t\right)+V_{2}\left(x_{2}, t\right)+V_{1} 2\left(x_{1}-x_{2}\right)
$$

Las dos cabezas de la kinesina están en contacto mediante una interacción elástica no lineal dada por:

$$
V_{12}(x)=-\frac{1}{2} K R_{0}^{2} \ln \left(1-\frac{\left(x-l_{0}\right)^{2}}{R_{0}^{2}}\right)
$$

La representación de las curvas de nivel del potencial $V_{1}\left(x_{1}, t\right)$ en dos dimensiones es la siguiente:

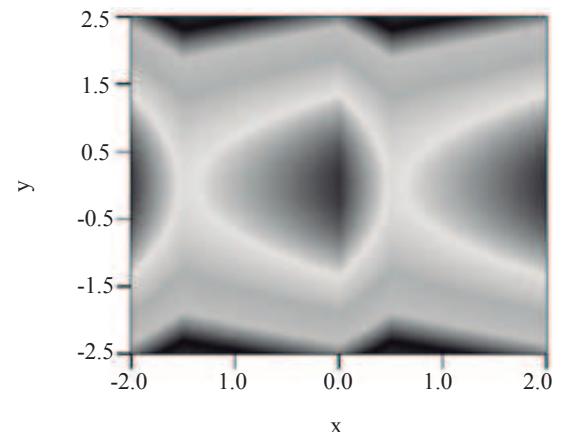

Figura 9. Representación de las curvas de nivel del potencial $V_{1}$ en 2D, los valles corresponden a $x=2 n$ con $(n=0, \pm 1, \ldots)$ y $y=0$. (figura tomada de [2]).

De forma análoga al análisis realizado por los paisajes de energía de la máquina macroscópica, puede ser analizado el potencial de la figura 9. En los valles (zonas oscuras) del paisaje de energía se puede establecer un camino unidimensional por el cual una de las cabezas de la kinesina se transporta. El potencial $V_{2}$ es el mismo, solamente que desplazado $l_{0}$, y mostraría la trayectoria para la segunda partícula. Dada la periodicidad en el potencial y el continuo movimiento de la kinesina, se puede establecer un ciclo en los pasos dados por esta, el cual está representado por:

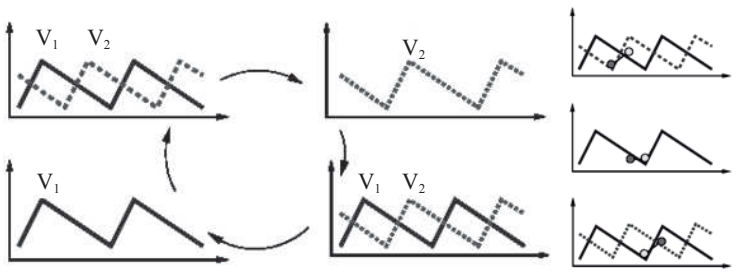

Figura 10. Representación del ciclo de dos pasos dados por la kinesina con movimiento hand-over-hand bajo un potencial flashing ratchet [2].

En el primer paso $(t=0)$, ambas partículas están confinadas cerca de los mínimos de su respectivo potencial, separadas por una distancia $l_{0}$ (distancia natural del cuello). Después de un tiempo, el potencial se encuentra en estado $t=t_{\text {on }}+t_{\text {off }}$, es decir, se apaga uno de los potenciales, lo que genera que una 
de las partículas se difunda almacenando energía, mientras que la otra cae a su mínimo. Después de un tiempo se enciende el potencial, lo que genera que la partícula antes libre se deslice y conserve la distancia $l_{0}$ con su compañera, esta parte del ciclo es un símil del primer paso. Nuevamente pasa un tiempo $t=t_{o f f}+t_{o n}$ y la partícula antes confinada es la que se difunde, mientras que la primera ya ha dado un paso, puesto que la que antes almacenaba energía se encuentra ahora en el siguiente mínimo, mientras que la otra ahora se difunde y almacena energía. El potencial es nuevamente encendido, lo que produce que las partículas estén ahora confinadas en el siguiente mínimo de su respectivo potencial y se cierre el ciclo con un paso para cada una de las partículas.

Así, este sistema puede ser modelado con la ecuación de Langevin bajo un potencial tipo flashing ratchet y explicar el movimiento Hand-overhand de una kinesina bicéfala transportándose en un microtúbulo.

\section{Conclusiones}

En este trabajo hemos estudiado algunos aspectos de la rectificación de fluctuaciones térmicas por medio de motores brownianos y del efecto ratchet, partiendo del teorema de fluctuación disipación de partículas con movimiento aleatorio o browniano. Por otra parte, modelamos la ecuación de Langevin con un potencial tipo flashing, para así aplicarlo a un modelo biológico a escala molecular, conocido como motor molecular, el cual explica el transporte proteico dirigido en el interior de una célula eucariota.

Los análisis más importantes del trabajo y las principales conclusiones que se derivan de ellos, se resumen a continuación.

A nivel nanoscópico, las fluctuaciones térmicas son las que rigen este mundo, así que un sistema inmerso en un fluido a una temperatura dada producirá que la dispersión de posición y velocidad de sus partículas generen grandes fluctuaciones en estas y a su vez se produzca una difusión al azar; esto también hace que la trayectoria de las partículas sean observables a escalas mayores (como lo observado por Brown). Aunque el movimiento de una partícula de este sistema es impredecible, su difusión puede ser calculada, puesto que obedece a una ecuación sencilla, dada por la relación de Einstein, $\eta D=k T$. Si al calcular el valor de $k_{B}$ para varios tipos de partículas, de varios tamaños, a varias temperaturas, en este sistema encontramos que efectivamente se obtiene lo que hace que esta ley se cumpla. Si además alejamos el sistema del equilibrio termodinámico bajo ciertas condiciones sin violar ninguna ley termodinámica, producirá que la difusión no sea al azar, sino que sea más probable en una dirección, generando así un movimiento rectificado o dirigido, que es conocido como efecto ratchet.

Por otro lado, las células están llenas de estructuras localizadas; algunos lugares actúan como "fábricas", cuyos productos deben ser transportados a sus "clientes" distantes. Vimos en el transcurso del trabajo que este fenómeno de transporte se realiza mediante algún tipo de motor molecular, cuya explicación quiso realizarse y modelarse bajo fenómenos físicos; por tal razón, analizamos el movimiento térmico (cuya importancia en el mundo nanoscópico la acabamos de destacar), ya que es, en cierta manera, el responsable del transporte molecular. Dicho análisis se realizó bajo las ecuaciones de Langevin, con ruido térmico blanco (pues es el encargado de que las partículas fluctúen sin dirección preferencial), junto con un potencial de dientes de sierra que hace que el sistema se desequilibre y pierda simetría; sumados estos dos, produce el efecto ratchet. Así, podemos concluir que la ecuación de Langevin es capaz de modelar y predecir el transporte dirigido de partículas brownianas alejadas del equilibrio térmico.

Se modelaron también un par de máquinas mecánicas capaces de extraer trabajo de un baño térmico y utilizarlo de forma rectificada, esto sin violar las leyes de la termodinámica. La razón fue sencilla: tratar de explicar el fenómeno de transporte desde el punto de vista termodinámico y estadístico de algo observable, para poderlo aplicar a escala nanométrica. Analizamos que de dicha máquina podíamos obtener un paisaje de energía potencial, pero no de energía cinética, porque la disipación viscosa es grande en el mundo nanoscópico, y concluimos que las máquinas a escala molecular se desplazan caminando aleatoriamente por su paisaje de energía y pueden atravesar barreras debido a que almacenan energía potencial, convirtiéndose en movimiento direccional, si es estructuralmente asimétrica. Pero nos dimos 
cuenta de que la asimetría estructural del sistema no es suficiente, por sí sola, para que este avance, puesto que ella no es dirigida si está en equilibrio termodinámico; para obtener trabajo útil es necesario llevarla fuera del equilibrio, lo que supone un paisaje de energía libre inclinado.

Lo anterior explica cómo era el transporte dirigido de moléculas proteicas como la kinesina, pero esto no explica el cómo caminaba, así que se intentó modelar la ecuación de Langevin con dos potenciales tipo flashing ratchet que daban a entender un tipo de movimiento llamado hand-over-hand con ciertos parámetros como la interacción entre las cabezas de la proteína, la distancia natural entre estas y el tiempo de prendido y apagado de los potenciales. Se cree que este modelamiento funciona adecuadamente y predice el movimiento de una kinesina transportando una carga aplicada por un microtúbulo y con movimiento tipo hand-over-hand.

\section{Referencias}

[1] R. P. Feynman, R. B. Leighton, and M. Sands, The Feynman Lectures on Physics, Mainly Mechanics, Radiations and Heat, Volume I. Addison-Wesley Publishir Company, 1963.

[2] J. Munárriz, J. J. Mazo, and F. Falo, "Model for hand-over-hand motion of molecular motors", Physical Review Letters E, 77, 031915, 2008.

[3] A. Einstein, Investigations on the Theory of the Brownian Movement, 1905.

[4] N. Philip, Física Biológica. Energía, Información, Vida, Editorial Reverté, 2005.

[5] C. J. Adkins, Equilibrium Thermodynamics. McGraw Hill Book Company, Cambridge University, Thitd Edition, 1983.

[6] K. Huang, Statistical Mechanics, 2 Ed., Wiley, 1987.

[7] R. Paniagua, Biologia Celular, Cap. 6: "Citoesqueleto”, Editorial McGraw Hill, 2007.

[8] R. K. Pathria. Statistical Mechanics, 2 Ed., Butterworth-Heinemann, 1996.
[9] F. Reif. Fundamentals of statistical and thermal physics. McGraw Hill Book Company.

[10] F. Reif. Física Estadística, Berkeley physics course volumen 5. Editorial Reverté. 1993.

[11] M. W. Zemansky, R.H. Dittman, Calor y Termodinámica, McGraw Hill Company, Sexta Edición, 1986.

[12] P. Reimann, Brownian motors: noisy transport far from equilibrium. Institut für Physik, Universität Augsburg, Universitätsstr. 1, 86135 Augsburg, Germany.

[13] P. Reimann, P. Häanggi, "Introduction to the physics of Brownian motors". Universität Augsburg, Universitätsstr. 1, 86135 Augsburg, Germany, Appl. Phys. A, 75, 169-178, 2002.

[14] M. J. Schnitzer, K. Visscher. S. M. Block, "Force production by single kinesin motors", Letters to Nature Cell Biology, vol. 2, pp. 718-723.

[15] Torsten Flue $\beta$ bach, Curso de Física Estadística, Fundacion Colouste Gulbenkian, 1999.

[16] J. Munárriz, J. J. Mazo, and F. Falo, "Model for hand-over-hand motion of molecular motors", Physical Review Letters, 77, 2010.

[17] Prweb, [online], Disponible en: http://ww1. prweb.com/prfiles/2009/09/08/140163/XVIVO HarvardKinesinMidView.jpg

[18] R. Dean Astumian, "Thermodynamics and Kinetics of a Brownian Motor", Science, vol. 276, May, 1997.

[19] A. Yildiz, M. Tomishige, R.D. Vale, P. R. Selvin. "Kinesin Walks Hand-Over-Hand", Science, vol. 303, pp. 676-678, 2005.

[20] C. L. Asbury, A. N. Fehr, S. M. Block. "Kinesin Moves by an Asymmetric Hand-Over-Hand Mechanism”, Science, vol. 302, pp. 2130-2134, 2003.

[21] K. Visscher, M. J. Schnitzer, S. M. Block. "Single kinesin molecules studied with a molecular force clamp", Letters to Nature Cell Biology, vol. 400, pp. 184-189. 